\title{
The Genetics and Biology of Drosophila
}

Volumes 1a, 1b, 1c

edited by M. Ashburner Department of Genetics, University of Cambridge, England

and E. Novitski Department of Biology, University of Oregon, Eugene, Oregon, USA

Volume 1a

April/May 1976, xx+530pp., £16.80/\$41.75 0.12.064901.2

Volume 1b

May/June 1976, xx+504pp., f00.00/\$00.00 0.12.064902.0

Volume 1c

June/July 1976, xx+510pp., f00.00/\$00.00 0.12.064903.9

Studies with Drosophila, in particular with Drosophila melanogaster, have contributed more to our understanding of heredity than studies with any other eukaryotes. It is genetically the best known "higher" organism; this means not only that a greater number of mutations are known in Drosophila than in other organisms, but also that we have an unrivalled ability to manipulate its chromosomes and mutations to experimental advantage. Drosophila is the organism of choice for studies of development, behaviour and evolution as well as the formal mechanisms of genetics. Current research ranges from problems in "pure genetics" to sophisticated studies in neurobiology and ecology.

Surprisingly, this is the first attempt since 1925 to publish a comprehensive account of the biology and genetics of Drosophila. It aims to collate the dauntingly large literature on the subject and to make more accessible the private language ot the Drosophilist. In this way the work will be of value both to established research workers and to biologists in general. This remarkable and ambitious work has been divided into three series of volumes. Volumes $1 \mathrm{a}, 1 \mathrm{~b}$ and $1 \mathrm{c}$ deal with the formal genetics of Drosophila, Volume 2 deals with its biology and development and Volume 3 with its evolution and ecology.

These volumes will prove an invaluable source of reference for Drosophilists, advanced students of genetics and for biologists, entomologists and zoologists with an interest in Drosophila research.

\section{Academic Press}

\section{London New York San Francisco}

A Subsidiary of Harcourt Brace Jovanovich, Publishers

24-28 Oval Road, London NW1, England

111 Fifth Avenue, New York, NY 10003, USA

Australian office: PO Box 300, North Ryde, NSW 2113, Australia

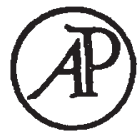

